\title{
UK Biobank's cardiovascular magnetic resonance protocol
}

\author{
Steffen E. Petersen 1*, Paul M. Matthews ${ }^{2}$, Jane M. Francis ${ }^{3}$, Matthew D. Robson³, Filip Zemrak', Redha Boubertakh', \\ Alistair A. Young ${ }^{4}$, Sarah Hudson ${ }^{5}$, Peter Weale ${ }^{6}$, Steve Garratt ${ }^{5}$, Rory Collins ${ }^{5}$, Stefan Piechnik ${ }^{3}$ \\ and Stefan Neubauer ${ }^{3}$
}

\begin{abstract}
Background: UK Biobank's ambitious aim is to perform cardiovascular magnetic resonance (CMR) in 100,000 people previously recruited into this prospective cohort study of half a million 40-69 year-olds.

Methods/design: We describe the CMR protocol applied in UK Biobank's pilot phase, which will be extended into the main phase with three centres using the same equipment and protocols. The CMR protocol includes white blood CMR (sagittal anatomy, coronary and transverse anatomy), cine CMR (long axis cines, short axis cines of the ventricles, coronal LVOT cine), strain CMR (tagging), flow CMR (aortic valve flow) and parametric CMR (native T1 map).

Discussion: This report will serve as a reference to researchers intending to use the UK Biobank resource or to replicate the UK Biobank cardiovascular magnetic resonance protocol in different settings.
\end{abstract}

\section{Background}

Understanding the determinants of diseases, such as myocardial infarction and stroke, is critical to advance medical knowledge that can lead to prolongation of life and improvement in quality of life. A combination of various risk factors often leads to disease with each risk factor having only moderate effects that also interact with each other in complex ways. Prospective cohort studies allow insights into risk factors before disease develops or into how disease management affects participants.

\section{Methods/Design}

UK Biobank, a prospective cohort study of half a million 40-69 year-olds (mean age 56.5 years, $54.4 \%$ female, 94.4 \% White, 1.9 \% Asian/Asian British, $1.6 \%$ Black/ Black British), started to recall participants for a comprehensive imaging visit. Baseline summary characteristics of the cohort can be viewed in the data showcase on UK Biobank's Web site (www.ukbiobank.ac.uk).

\footnotetext{
* Correspondence: s.e.petersen@qmul.ac.uk

${ }^{1}$ William Harvey Research Institute, NIHR Cardiovascular Biomedical Research Unit at Barts, Queen Mary University of London, Charterhouse Square, London EC1M 6BQ, UK

Full list of author information is available at the end of the article
}

The imaging visit includes a 35-min brain magnetic resonance imaging (MRI) scan at 3 Tesla, a dual energy $\mathrm{X}$ -ray absorptiometry (DEXA) scan (10-15 min), and carotid ultrasound (10-15 $\mathrm{min}$ ) in addition to preparation (including consenting) and collection of non-imaging data and biological samples (e.g. partial repeat of the baseline assessment visit with supplementary cognitive function tests), and 20-min cardiovascular magnetic resonance (CMR) at 1.5 Tesla and 10-min abdominal MRI also at 1.5 Tesla (average time over 3 month period was $30 \mathrm{~min}$ for both parts combined at $1.5 \mathrm{~T}$ Tesla). This resulted in an average total visit time of $3 \mathrm{~h} 29$ mins.

The rationale, challenges and approaches to perform 100,000 CMR scans in UK Biobank have been described elsewhere [1]. The pilot phase is almost completed with more than 7300 participants as of early January 2016 (see the current subject counter displayed on www.ukbiobank.ac.uk). A first release of these imaging (with associated phenotypic) data occurred in October 2015.

The purpose of this article is to describe the details of the relevant methodology for CMR acquisition during the first 20 months of the pilot phase, which started in May 2015. This article aims to serve researchers as a source of information for planning access applications (www.ukbiobank.ac.uk/register-apply/) to this shared 
resource and to serve as a reference for publications arising from the use of UK Biobank CMR data.

The CMR protocol was developed bearing in mind the set-up and requirements described previously [1]. In brief, each participant undergoes a 20-min CMR protocol without pharmacological stressor or contrast agent, as part of a 30- min combined CMR and abdominal MRI protocol.

\section{Cardiovascular magnetic resonance infrastructure}

CMR imaging is being performed in Cheadle, United Kingdom, on a clinical wide bore 1.5 Tesla scanner (MAGNETOM Aera, Syngo Platform VD13A, Siemens Healthcare, Erlangen, Germany). The scanner is equipped with 48 receiver channels, a $45 \mathrm{mT} / \mathrm{m}$ and $200 \mathrm{~T} / \mathrm{m} / \mathrm{s}$ gradient system, an 18 channels anterior body surface coil used in combination with a 12 elements of an integrated 32 element spine coil and electrocardiogram (ECG) gating for cardiac synchronization.

In addition to the vendor's advanced cardiac package, the Shortened Modified Look-Locker Inversion recovery technique (ShMOLLI, WIP780B) is implemented on the scanner in order to perform native (non-contrast) myocardial T1 mapping. The Cardiac Dot Engine (Siemens Healthcare, Erlangen, Germany) is used to facilitate quality for consistency of image acquisition throughout the study.

\section{CMR protocol}

UK Biobank's CMR acquisitions include piloting and sagittal, transverse and coronal partial coverage of the chest and abdomen. For cardiac function, three long axis cines (horizontal long axis - HLA, vertical long axis VLA, and left ventricular outflow tract -LVOT cines both sagittal and coronal) and a complete short axis (SA) stack of balanced steady state free precession (bSSFP) cines, covering the left ventricle (LV) and right ventricle (RV) are acquired (Fig. 1). Aortic compliance can be derived from a transverse bSSFP cine at the level of the pulmonary trunk and right pulmonary artery (Fig. 2). Immediately before and after this bSSFP acquisition of the aorta, brachial blood pressure readings are being obtained using a manual sphygmomanometer used for calibrating peripheral waveforms and immediately afterwards a brachial pressure wave trace is digitally computed by the Vicorder (Skidmore Medical, Bristol, UK) with the cuff statically inflated to $70 \mathrm{mmHg}$ using a volume displacement technique. The Vicorder software calculates values for central blood pressure by applying a previously described brachial-to-aortic transfer function [2]. Aortic distensibility represents the relative change in area of the aorta per unit pressure, taken here as the central pulse pressure and is calculated according to the formula:

$$
\text { aortic distensibility }=\left(\mathrm{A}_{\max } \mathrm{A}_{\min }\right) / \mathrm{A}_{\min } /\left(\mathrm{P}_{\max } \mathrm{P}_{\min }\right) \text {, }
$$

where $\mathrm{A}_{\max }=$ maximal (systolic) area $\left(\mathrm{mm}^{2}\right), \mathrm{A}_{\min }=\min$ imal (diastolic) area $\left(\mathrm{mm}^{2}\right), \mathrm{P}_{\max }=$ systolic blood pressure $(\mathrm{mm} \mathrm{Hg})$, and $\mathrm{P}_{\min }=$ diastolic blood pressure $(\mathrm{mm}$ $\mathrm{Hg})$ [3].

A phase contrast sequence is planned on both sagittal and coronal LVOT cines to capture aortic flow and the number of valve cusps (Fig. 3). The plane is located at or immediately above the sino-tubular junction at end diastole as recommended [4]. The standard velocity encoding (VENC) is $2 \mathrm{~m} / \mathrm{s}$ but is adjusted upwards based on presence/degree of turbulence seen on the LVOT cines and if time allows. Tagging (grid) is acquired in 3 short axis views (basal, midventricular and apical) carefully avoiding the LVOT in the basal slice. The midventricular slice position of the tagging matches the native T1 mapping short axis slice. An international expert advisory group helped to guide adjustments made to the originally planned protocol [1] to ensure that the protocol could be consistently conducted within $20 \mathrm{~min}$, but these have not compromised the information available.

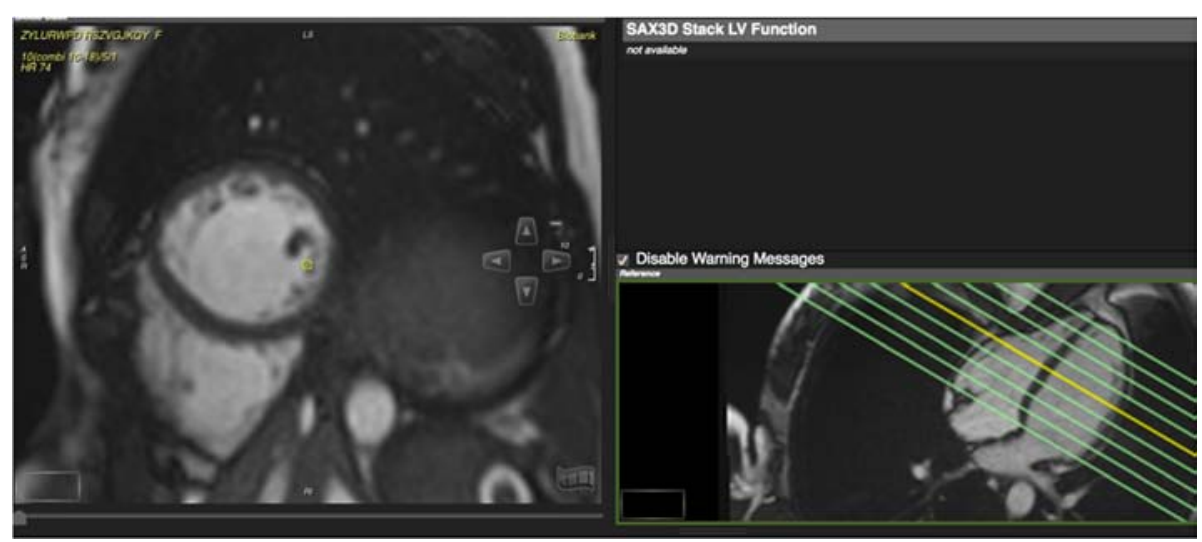

Fig. 1 Planning of the short axis cine stack covering the entire left and right ventricles 


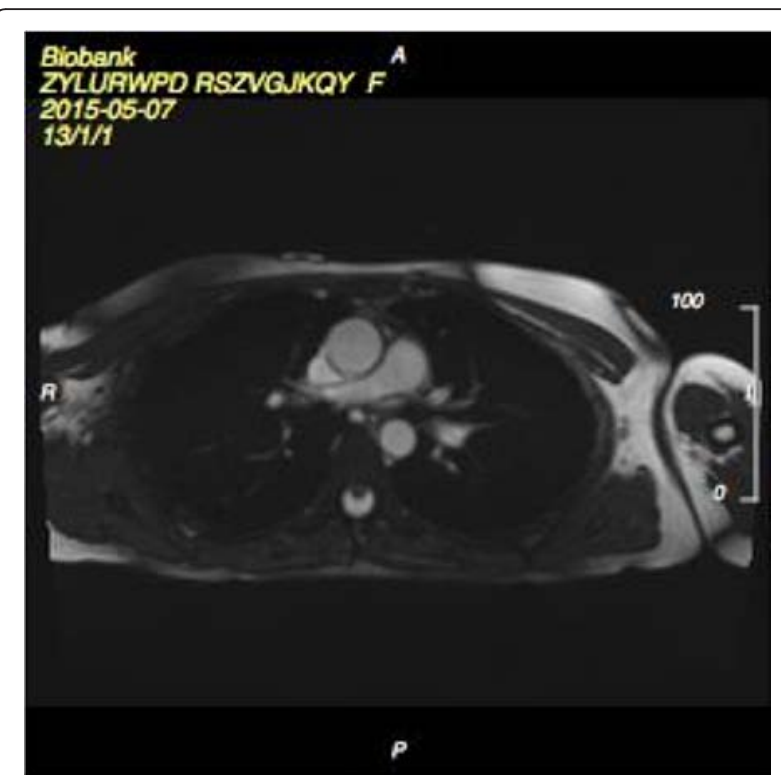

Fig. 2 Transverse aortic cine at the level of the pulmonary trunk/ right pulmonary artery

The atrial short axis cine stack has been removed; atrial end-diastolic and end-systolic volumes and atrial ejection fraction can be measured with the long-axis cines. Three long-axis tagged cines have been removed to save time, but the radial and longitudinal strain parameters can be derived reliably using feature-tracking techniques from the untagged long-axis cines acquired. A phase contrast sequence $(\sim 1 \mathrm{~min})$ was added to allow aortic flow and number of aortic cusps to be captured, adding scientific value, since aortic stenosis is the most common heart valve lesion, has a poor prognosis if severe and is increasingly common in ageing populations. Also native T1 mapping in one midventricular short axis was added to allow myocardial tissue characterisation without the use of contrast agents. The details of the CMR sequences are summarised in Table 1.

\section{CMR image analysis}

Since the time of first data release, researchers are able to access the DICOM CMR image files, but only a limited range of measures derived from images is currently available. The automated inline ventricular function option is enabled on the scanner, which provides automatic assessment of LV contours and volumes. Given that UK Biobank provides LV end-diastolic volume, LV endsystolic volume, LV stroke volume, cardiac output and cardiac index to researchers without checking the endocardial and epicardial contours for quality it may be recommended that these are quality checked as the data application requires. A British Heart Foundation (BHF) project grant (PG/14/89/31194, PI Petersen, 6/2015 to $5 / 2018$ ) currently funds the manual analysis to create a CMR reference standard for the UK Biobank imaging resource in 5000 CMR scans. Table 2 provides an updated list of derived CMR parameters that will be returned to the UK Biobank resource upon completion of the analysis. A UK Biobank CMR Image Analysis Consortium has been formed and has been planning issues around standardization and automating CMR image analysis. The work of the consortium expects to reduce the overall costs associated with cardiovascular image analysis for large-scale cohort studies through knowledge sharing, coordination of efforts and capacity building. An important aspect of the user data access agreement with UK Biobank is that measures derived in the course of individual research projects that are of general value to the research community will be returned to UK Biobank to be incorporated into the database (with a full description of the methods used) for others in the research community to access after an appropriate embargo period. Through this mechanism, for example, the CMR Image Analysis Consortium will return to the UK Biobank

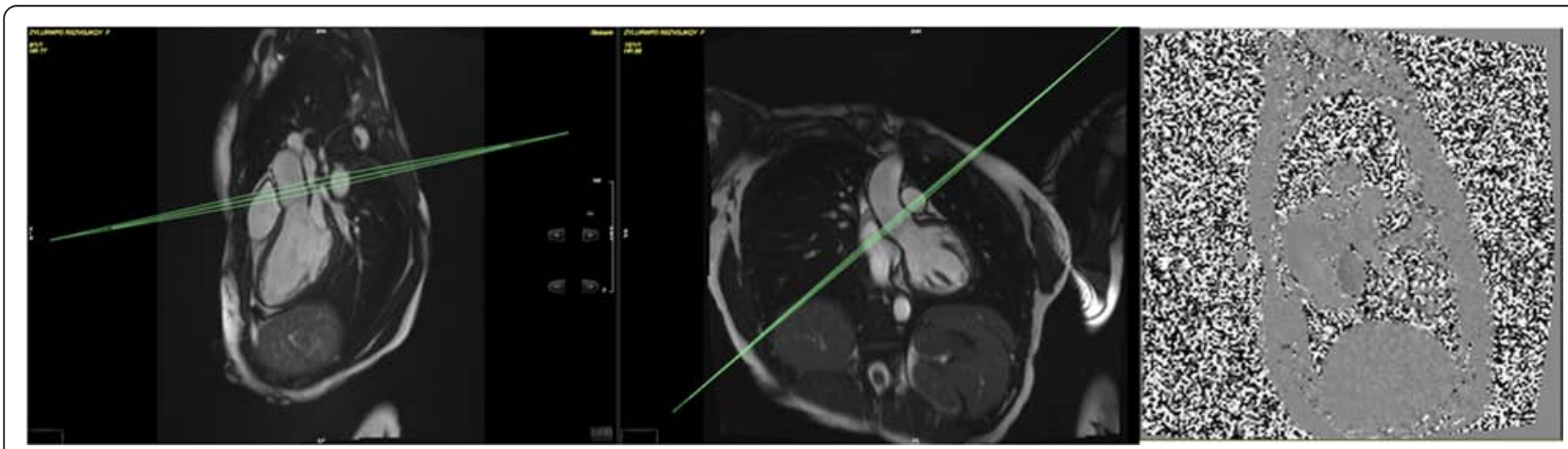

Fig. 3 Aortic valve flow imaging view planned using the sagittal and coronal left ventricular outflow tract (LVOT) cines 
Table 1 Cardiovascular magnetic resonance protocol for UK Biobank

\begin{tabular}{|c|c|c|c|c|c|c|c|c|c|}
\hline Description & $\begin{array}{l}\text { Sagittal } \\
\text { anatomy }\end{array}$ & $\begin{array}{l}\text { Coronal and } \\
\text { Transverse } \\
\text { anatomy" }\end{array}$ & Long axis cines & Short axis cines & $\begin{array}{l}\text { Aortic distensibility } \\
\text { cine }\end{array}$ & Tagging & $\begin{array}{l}\text { Coronal } \\
\text { LVOT cine }\end{array}$ & $\begin{array}{l}\text { Aortic valve } \\
\text { flow }\end{array}$ & Native $T 1$ map \\
\hline $\begin{array}{l}\text { Pulse sequence: } \\
\text { Simplified terminology } \\
\text { (scientific terminology) }\end{array}$ & $\begin{array}{l}\text { White blood } \\
\text { CMR (bSSFP) }\end{array}$ & $\begin{array}{l}\text { White blood } \\
\text { CMR(bSSFP) }\end{array}$ & $\begin{array}{l}\text { Cine CMR } \\
\text { (bSSFP) }\end{array}$ & $\begin{array}{l}\text { Cine CMR } \\
\text { (bSSFP) }\end{array}$ & $\begin{array}{l}\text { Cine CMR } \\
\text { (bSSFP) }\end{array}$ & $\begin{array}{l}\text { Strain } \\
\text { CMR(GRE) }\end{array}$ & $\begin{array}{l}\text { Cine CMR } \\
\text { (bSSFP) }\end{array}$ & $\begin{array}{l}\text { Flow CMR } \\
\text { (GRE) }\end{array}$ & $\begin{array}{l}\text { Parametric } \\
\text { CMR (ShMOLLI } \\
\text { WIP780B) }\end{array}$ \\
\hline Flip angle $\left(^{\circ}\right)$ & 80 & 80 & 80 & 80 & 80 & 12 & 80 & 20 & 35 \\
\hline TR (ms) & 2.6 & 2.6 & 2.7 & 2.6 & 2.8 & 8.2 & 2.7 & 4.6 & 2.6 \\
\hline TE (ms) & 1.12 & 1.12 & 1.16 & 1.10 & 1.17 & 3.90 & 1.16 & 2.47 & 1.07 \\
\hline GRAPPA factor & 2 & 2 & 2 & 2 & 2 & 0 & 2 & 2 & 2 \\
\hline Slice thickness (mm) & 8.0 & 8.0 & 6.0 & 8.0 & 6.0 & 8.0 & 6.0 & 6.0 & 8.0 \\
\hline Slice gap (mm) & 2.64 & 4 & n.a. & 2 & n.a. & n.a. & n.a. & n.a. & n.a. \\
\hline $\begin{array}{l}\text { Typical Field of } \\
\text { View }(\mathrm{mm})\end{array}$ & $400 \times 400$ & $400 \times 400$ & $380 \times 274$ & $380 \times 252$ & $380 \times 294$ & $350 \times 241$ & $380 \times 384$ & $340 \times 340$ & $360 \times 236$ \\
\hline Matrix & $240 \times 158$ & $240 \times 158$ & $208 \times 187$ & $208 \times 187$ & $240 \times 216$ & $256 \times 174$ & $208 \times 187$ & $192 \times 173$ & $192 \times 192$ \\
\hline Voxel size & $1.7 \times 1.7 \times 8.0$ & $1.7 \times 1.7 \times 8.0$ & $1.8 \times 1.8 \times 6.0$ & $1.8 \times 1.8 \times 8.0$ & $1.6 \times 1.6 \times 6.0$ & $1.4 \times 1.4 \times 8.0$ & $1.8 \times 1.8 \times 6.0$ & $1.8 \times 1.8 \times 6.0$ & $\begin{array}{l}0.9 \times 0.9 \times 8.0 \\
\text { (Interpolation }= \\
\text { On, factor } 2)\end{array}$ \\
\hline $\begin{array}{l}\text { Acquired temporal } \\
\text { resolution (ms) }\end{array}$ & n.a. & n.a. & 32.64 & 31.56 & 28.00 & 41.05 & 32.64 & 37.12 & 368.28 \\
\hline $\begin{array}{l}\text { Calculated } \\
\text { cardiac phases }\end{array}$ & 1 & 1 & 50 & 50 & 50 & 1 & 50 & 30 & 1 \\
\hline $\begin{array}{l}\text { ECG triggering/ } \\
\text { gating }\end{array}$ & PT & n.a. & $R G$ & $R G$ & RG & PT & RG & $R G$ & PT \\
\hline $\begin{array}{l}\text { Other } \\
\text { parameters }\end{array}$ & & & $\begin{array}{l}\text { Inline } \\
\text { Evaluation } \\
\text { Ventricular } \\
\text { Function }\end{array}$ & $\begin{array}{l}\text { Inline Evaluation } \\
\text { Ventricular Function }\end{array}$ & & $\begin{array}{l}\text { Grid spacing } \\
6 \mathrm{~mm} \text {, shared } \\
\text { phases }\end{array}$ & & & $\begin{array}{l}\text { T1 map } \\
\text { determined } \\
\text { on-line. }\end{array}$ \\
\hline Image Filter & Off & Off & Off & Off & Off & Off & Off & Off & Off \\
\hline Distortion Corr & On (2D) & On (2D) & On (2D) & On (2D) & On (2D) & On (2D) & On (2D) & On (2D) & Off \\
\hline Raw Filter & Off & Off & Off & Off & Off & Off & Off & Off & $\begin{array}{l}\text { On: Weak, } \\
\text { slope } 25\end{array}$ \\
\hline Elliptical filter & Off & Off & Off & Off & Off & Off & Off & Off & Off \\
\hline
\end{tabular}


Table 1 Cardiovascular magnetic resonance protocol for UK Biobank (Continued)

\begin{tabular}{|c|c|c|c|c|c|c|c|c|c|}
\hline $\begin{array}{l}\text { No of } \\
\text { breath-holds } \\
\text { (expiration) }\end{array}$ & 1 & 1 & $\begin{array}{l}1 \text { slice per } \\
\text { breath-hold }\end{array}$ & $\begin{array}{l}1 \text { slice per } \\
\text { breath-hold }\end{array}$ & 1 & $\begin{array}{l}1 \text { slice per breath- } \\
\text { hold }\end{array}$ & 1 & 1 & 1 \\
\hline Orientation & $\begin{array}{l}\text { Sagittal } \\
(\times 11) P E \\
\text { direction = AP }\end{array}$ & $\begin{array}{l}\text { Coronal }(\times 10) \\
\text { Transverse } \\
(\times 10) P E \text { direction }= \\
\text { RL \& AP }\end{array}$ & $\begin{array}{l}\text { HLA, VLA, } \\
\text { LVOT (sagittal) } \\
\text { viewsPE } \\
\text { direction = varies }\end{array}$ & $\begin{array}{l}\text { Coverage based } \\
\text { to apex in SA } \\
\text { views (approximately x10) } \\
\text { PE direction = AP }\end{array}$ & $\begin{array}{l}\text { Transverse at level } \\
\text { of pulmonary } \\
\text { trunk/right } \\
\text { pulmonary } \\
\text { arteryPE } \\
\text { direction = AP }\end{array}$ & $\begin{array}{l}\text { SA views } \\
(b, m, a) P E \\
\text { direction = AP }\end{array}$ & $\begin{array}{l}\text { LVOT } \\
\text { (coronal) } \\
\text { view PE } \\
\text { direction = RL }\end{array}$ & $\begin{array}{l}\text { Aortic valve plane } \\
\text { planned on both } \\
\text { LVOT cinesPE } \\
\text { direction = AP }\end{array}$ & $\begin{array}{l}\text { SA }(m) P E \\
\text { direction = AP }\end{array}$ \\
\hline Image example & & & & & & & & & \\
\hline
\end{tabular}

Abbreviations: bSSFP balanced steady state free precession, PT Prospective Triggering, RG Retrospective Gating, $b$ basal, $m$ midventricular, $a$ apical, HLA Horizontal long axis, VLA Vertical Long Axis, LVOT Left Ventricular Outflow Tract, SA Short Axis 
Table 2 The minimum dataset from the CMR images analyzed manually in the first 5,000 CMR scans will be returned to the UK Biobank resources and will likely include

\begin{tabular}{|c|c|}
\hline $\begin{array}{l}\text { Cardiovascular } \\
\text { structure }\end{array}$ & Quantifiable parameters \\
\hline Left ventricle & $\begin{array}{l}\text { Myocardial mass (g), ejection fraction (\%), end-diastolic } \\
\text { volume (ml), end-systolic volume (ml), stroke volume } \\
(\mathrm{ml}) \text { and the corresponding values indexed to body } \\
\text { surface area, height or weight. Time to peak contraction } \\
\text { and filling rates (both in s) and peak contraction and } \\
\text { filling rates (both in } \mathrm{ml} / \mathrm{s}) \text { can be derived from the cine } \\
\text { images accepting the limitation of limited temporal } \\
\text { resolution for this purpose. American Heart Association } \\
\text { (AHA) myocardial segments: end-diastolic thickness } \\
\text { (mm), end-systolic thickness (mm), thickening (mm), } \\
\text { thickening (\%). Strain (\%) and strain-rates (\%/s) in three } \\
\text { directions (radial [ERR], circumferential [ECC] and } \\
\text { longitudinal [ELL]) and in systole and diastole and } \\
\text { corresponding changes in angle caused by shear } \\
\text { (ERC and ECL). Torsion-rates (degrees/s). Midwall } \\
\text { midventricular native T1 (ms). }\end{array}$ \\
\hline $\begin{array}{l}\text { Right } \\
\text { ventricle }\end{array}$ & $\begin{array}{l}\text { Myocardial mass }(\mathrm{g}) \text {, ejection fraction }(\%) \text {, end-diastolic } \\
\text { volume }(\mathrm{ml}) \text {, end-systolic volume }(\mathrm{ml}) \text {, stroke volume } \\
(\mathrm{ml}) \text { and the corresponding values indexed to body } \\
\text { surface area, height or weight }\end{array}$ \\
\hline Left atrium & $\begin{array}{l}\text { End-diastolic area ( } \mathrm{cm} 2) \text {, end-systolic area ( } \mathrm{cm} 2) \text { and } \\
\text { left atrial diameters as typically measured by 2D- } \\
\text { echocardiography approaches. Using the area length } \\
\text { formula atrial volumes (ml), atrial stroke volume (ml) } \\
\text { and atrial ejection fraction (\%) can be derived. }\end{array}$ \\
\hline Right atrium & $\begin{array}{l}\text { End-diastolic area (cm2), end-systolic area ( } \mathrm{cm} 2 \text { ) and } \\
\text { right atrial diameters as typically measured by 2D- } \\
\text { echocardiography approaches. Using the area length } \\
\text { formula atrial volumes (ml), atrial stroke volume (ml) } \\
\text { and atrial ejection fraction (\%) can be derived. }\end{array}$ \\
\hline Aorta & $\begin{array}{l}\text { Distensibility }(1 / \mathrm{mmHg}) \text { in ascending aorta and } \\
\text { proximal descending aorta; diastolic aortic dimensions } \\
\text { (cm2): aortic root (annulus, sinus of valsalva, sinutubular } \\
\text { junction) ascending aorta and proximal descending } \\
\text { aorta. Diameter of abdominal aorta as visualized. }\end{array}$ \\
\hline Aortic valve & $\begin{array}{l}\text { Peak velocity (cm/s), peak gradient }(\mathrm{mmHg}) \text {, forward } \\
\text { volume }(\mathrm{ml}) \text {, regurgitant volume }(\mathrm{ml}) \text {, regurgitant } \\
\text { fraction }(\%) \text {. Number of cusps of aortic valve (bicuspid, } \\
\text { tricuspid, quadricuspid). }\end{array}$ \\
\hline
\end{tabular}

resource the manually segmented cardiac data and measures of the quality of the inline ventricular function contours expressed as calibration corrections for the automated tools relative to the manual analyses.

It is likely that different groups requesting access to UK Biobank will analyse images differently. Given this background we will not provide a detailed description of CMR image analysis approaches in this manuscript.

\section{Discussion}

We describe the CMR protocol applied in UK Biobank's pilot phase which will also be applied when UK Biobank extends this into the main phase with three centres using the same equipment and protocols. This manuscript will serve as a reference to researchers intending to use the UK Biobank resource for cardiac analyses or those who wish to replicate the UK Biobank CMR protocol in other settings.

\section{Abbreviations}

bSSFP: Balanced steady state free precession; BHF: British Heart Foundation: CMR: Cardiovascular magnetic resonance; DEXA: Dual energy $X$-ray absorptiometry; ECG: Electrocardiogram; HLA: Horizontal long axis; LV: Left ventricle; LVOT: Left ventricular outflow tract; RV: Right ventricle; SA: Short axis; VENC: Velocity encoding; VLA: Vertical long axis.

\section{Competing interests}

PMM has served as a consultant or received research funding from GlaxoSmithKline, Biogen, Novartis, IXICO and Adelphi Communications. SEP is Consultant to Circle Cardiovascular Imaging, Inc., Calgary, Canada. SN is a Consultant to Novartis, Cambridge, USA, and a Consultant, Shareholder and Non-Executive Director for Perspectum Diagnostics, Oxford, UK.

AAY is a consultant for Siemens Healthcare.

PW is an employee of Siemens Healthcare, Frimley, UK.

\section{Authors' contributions}

All authors 1) have made substantial contributions to conception and design 2) have been involved in drafting the manuscript or revising it critically for important intellectual content; and 3) have given final approval of the version to be published.

\section{Acknowledgements}

SEP was directly funded by the National Institute for Health Research Cardiovascular Biomedical Research Unit at Barts. SN acknowledges support from the Oxford NIHR Biomedical Research Centre and from the Oxford British Heart Foundation Centre of Research Excellence. SP and PL are funded by a BHF Senior Clinical Research fellowship. RC is supported by a BHF Research Chair and acknowledges the support of the Oxford BHF Centre for Research Excellence and the MRC and Wellcome Trust. AAY acknowledges support from the National Institutes of Health R01HL121754. PMM gratefully acknowledges relevant training fellowships and research support from the Wellcome Trust, the Medical Research Council, the Imperial College NIHR BRC. He gratefully acknowledges personal support from the Edmond J. Safra Foundation and Lily Safra. SEP, SN and SP acknowledge the British Heart Foundation (BHF) for funding the manual analysis to create a cardiovascular magnetic resonance imaging reference standard for the UK Biobank imaging resource in 5000 CMR scans (PG/14/89/31194, PI Petersen, 6/2015 to $5 / 2018$

\section{Author details}

${ }^{1}$ William Harvey Research Institute, NIHR Cardiovascular Biomedical Research Unit at Barts, Queen Mary University of London, Charterhouse Square, London EC1M 6BQ, UK. ${ }^{2}$ Division of Brain Sciences, Department of Medicine, Imperial College, London, UK. ${ }^{3}$ Division of Cardiovascular Medicine, Radcliffe Department of Medicine, University of Oxford, Oxford, UK. ${ }^{4}$ Department of Anatomy and Medical Imaging, University of Auckland, Auckland, New Zealand. '5K Biobank, Spectrum Way, Adswood, Stockport, Cheshire SK3 OSA, UK. ${ }^{6}$ Siemens Healthcare, Frimley, Surrey GU16 8QD, UK.

Received: 2 November 2015 Accepted: 22 January 2016

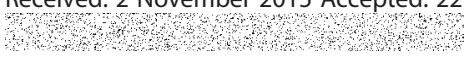

\section{References}

1. Petersen SE, Matthews PM, Bamberg F, Bluemke DA, Francis JM, Friedrich $M G$, et al. Imaging in population science: cardiovascular magnetic resonance in 100,000 participants of UK Biobank - rationale, challenges and approaches. J Cardiovasc Magn Reson. 2013;15:46.

2. O'Rourke MF. Influence of ventricular ejection on the relationship between central aortic and brachial pressure pulse in man. Cardiovasc Res. 1970;4:291-300. 
3. Rider OJ, Holloway CJ, Emmanuel Y, Bloch E, Clarke K, Neubauer S. Increasing plasma free fatty acids in healthy subjects induces aortic distensibility changes seen in obesity. Circ Cardiovasc Imaging. 2012:5:367-75.

4. Kilner PJ, Gatehouse PD, Firmin DN. Flow measurement by magnetic resonance: a unique asset worth optimising. J Cardiovasc Magn Reson. 2007;9:723-8.

Submit your next manuscript to BioMed Central and we will help you at every step:

- We accept pre-submission inquiries

- Our selector tool helps you to find the most relevant journal

- We provide round the clock customer support

- Convenient online submission

- Thorough peer review

- Inclusion in PubMed and all major indexing services

- Maximum visibility for your research

Submit your manuscript at www.biomedcentral.com/submit

) Biomed Central 